\title{
Cost-effectiveness of tenofovir/emtricitabine compared with other NRTI pairs in treatment-naïve adults with HIV infection in the USA
} AJ Brogan ${ }^{1}$, F Everhard ${ }^{2}$, SE Talbird ${ }^{1}$ and E Hutt*3

\author{
Address: ${ }^{1}$ RTI Health Solutions, Research Triangle Park, NC, USA, ${ }^{2}$ Gilead Sciences, Inc., Foster City, CA, USA and ${ }^{3}$ Gilead Sciences Europe Ltd., \\ Uxbridge, UK \\ * Corresponding author
}

from Ninth International Congress on Drug Therapy in HIV Infection

Glasgow, UK. 9-13 November 2008

Published: 10 November 2008

Journal of the International AIDS Society 2008, I I (Suppl I):P309 doi:I0.I I86/I758-2652-I I-SI-P309

This abstract is available from: http://www.jiasociety.org/content/II/SI/P309

(C) 2008 Brogan et al; licensee BioMed Central Ltd.

\section{Purpose of the study}

The efficacy and tolerability of nucleoside reverse transcriptase inhibitors (NRTIs) are well characterized. In this analysis we examined the cost-effectiveness of once-daily tenofovir/emtricitabine [TDF/FTC] compared with twicedaily zidovudine/lamivudine [ZDV/3TC] and once-daily abacavir/lamivudine $[\mathrm{ABC} / 3 \mathrm{TC}]$ in treatment-naïve HIVinfected adults in the US.

\section{Methods}

Lifetime costs and health outcomes for a treatment-naïve cohort were modeled using a Markov framework with six health states based on CD4 cell-count ranges. Efficacy data for first-line therapy were based on 144-week results of Study 934 comparing TDF/FTC + efavirenz [EFV] with ZDV/3TC + EFV and normalized 48-week results of Study CNA30024 comparing ABC/3TC + EFV with ZDV/3TC + $\mathrm{EFV}$. Immune response data were used to calculate transition probabilities between the CD4-based health states. Individuals with viral loads higher than 400 copies $/ \mathrm{mL}$ switched to second-line therapy at rates estimated over time using exponential functions fitted to trial data. Costs and efficacy for subsequent therapy lines were based on likely baskets of second-line, third-line, and non-suppressive therapy regimens. Costs for antiretroviral drugs were based on 2008 wholesale acquisition costs. Utility values, mortality rates, and costs for other medical resources were obtained from published sources. Adverse events observed in Studies 934 and CNA30024 were accounted for in modeled costs and outcomes. Base-case results were tested in one-way and probabilistic sensitivity analyses (PSA).

\section{Summary of results}

On average, individuals using TDF/FTC were predicted to remain on first-line therapy for 6.0 years, accrue total lifetime costs of $\$ 620,673$, and experience 14.22 qualityadjusted life-years (QALYs) compared with 4.9 years, $\$ 631,436$ and 13.89 QALYs for those using ZDV/3TC, and 5.0 years, $\$ 629,143$ and 14.10 QALYs for those using $\mathrm{ABC} / 3 \mathrm{TC}$. One-way sensitivity analysis predicted TDF/ FTC to be cost saving for almost all parameter ranges and scenarios tested; PSA showed TDF/FTC was likely to be cost saving.

\section{Conclusion}

TDF/FTC was predicted to be both more effective and cost saving compared with ZDV/3TC and ABC/3TC in treatment-naïve adults with HIV infection in the US. Cost savings were largely due to avoidance of more expensive subsequent lines of therapy and lower costs associated with adverse events and disease-related resource use. Results were robust to parameter uncertainty and modeling assumptions. 